\title{
Physical basis of colors seen in Congo red-stained amyloid in polarized light
}

\author{
Alexander J Howie ${ }^{1}$, Douglas B Brewer ${ }^{2}$, Daniel Howell ${ }^{3}$ and Adrian P Jones ${ }^{3}$
}

Amyloid stained by Congo red is traditionally said to show apple-green birefringence in polarized light, although in practice various colors may be seen between accurately crossed polarizing filters, called polarizer and analyzer. Other colors are seen as the polarizer and analyzer are uncrossed and sometimes when the slide is rotated. Previously, there has been no satisfactory explanation of these properties. Birefringence means that a material has two refractive indices, depending on its orientation in polarized light. Birefringence can change linearly polarized light to elliptically polarized, which allows light to pass a crossed analyzer. The birefringence of orientated Congo red varied with wavelength and was maximal near its absorption peak, changing from negative (slow axis of transmission perpendicular to smears or amyloid fibrils) on the shortwave side of the peak to positive (slow axis parallel) on the longwave side. This was explained by a property of any lightabsorbing substance called anomalous dispersion of the refractive index around an absorption peak. Negative birefringence gave transmission of blue, positive gave yellow, and the mixture was perceived as green. This explains how green occurs in ideal conditions. Additional or strain birefringence in the optical system, such as in glass slides, partly or completely eliminated blue or yellow, giving yellow/green or yellow, and blue/green or blue, which are commonly seen in practice and in illustrations. With uncrossing of polarizer or analyzer, birefringent effects declined and dichroic effects appeared, giving progressive changes from green to red as the plane of polarization approached the absorbing axis and from green to colorless in the opposite way. This asymmetry of effects is useful to pathologists as a confirmation of amyloid. Rather than showing 'apple-green birefringence in polarized light' as often reported, Congo red-stained amyloid, when examined between crossed polarizer and analyzer, should more accurately be said to show anomalous colors.

Laboratory Investigation (2008) 88, 232-242; doi:10.1038/labinvest.3700714; published online 31 December 2007

KEYWORDS: amyloid; anomalous colors; birefringence; Congo red; polarization

Many pathologists use Congo red to make a diagnosis of amyloid and state the common opinion that in polarized light, Congo red-stained amyloid shows apple-green birefringence, sometimes called apple-green dichroism. Is this opinion correct? A cursory glance at published micrographs said to illustrate this color reveals that most show more than one color and that some do not even show green. In everyday practice, a diligent pathologist may have been dissuaded from a diagnosis of amyloid, or at least puzzled, if green was not the only color or if green was not seen. If the general opinion is correct despite these findings and illustrations, what is the explanation of the green color? If it is not correct, what is the explanation of the colors seen, and what is a better expression to use? Does an understanding of the mechanisms help the pathologist in practice or give an insight into the interaction between Congo red and amyloid?
No previous explanation of the mechanisms has been satisfactory, partly because of incomplete accounts of the properties of Congo red-stained amyloid. We investigated these using polarizing microscopy. ${ }^{1-3}$ Because some principles of polarizing microscopy may not be widely known, they are given here to help understand this study.

\section{Polarizing Microscopy}

Polarization means that light waves vibrate in only one plane. One way to achieve this is by use of the polarizing filters available for microscopes, called a polarizer when inserted in the optical path between light source and specimen stage and an analyzer when inserted between the specimen stage and eyepieces. Polarization effects are generally identical if either filter is inserted on its own, or if either is rotated. If just a

\footnotetext{
${ }^{1}$ Department of Pathology, University College London, London, UK; ${ }^{2}$ Department of Pathology, University of Birmingham, Birmingham, UK and ${ }^{3}$ Department of Earth Sciences, University College London, London, UK

Correspondence: Professor AJ Howie, MD, Department of Cellular Pathology, Royal Free Hospital, London NW3 2QG, UK. E-mail: a.j.howie@medsch.ucl.ac.uk Received 3 September 2007; revised 21 November 2007; accepted 21 November 2007
} 
polarizer or an analyzer is inserted, without a specimen, the field appears bright, and the observer cannot detect that the light is polarized, even if the polarizing filter is rotated. If the other polarizing filter is also inserted, and one of them is rotated, the field now changes from light to dark, with maximal darkness when the planes of polarization of polarizer and analyzer are at right angles, and the filters are said to be crossed. When Congo red-stained amyloid is considered, this arrangement is what is usually meant by 'polarized light,' but a more accurate expression is examination of a specimen between crossed polarizer and analyzer. There are two observations to explain, namely, how Congo red-stained amyloid appears bright against a dark background under these conditions, and how it appears colored.

\section{Absorption and Dichroism}

Materials appear colored because they absorb light of certain wavelengths and their color is white lacking those wavelengths. The visible spectrum runs from violet at wavelengths of about $400-430 \mathrm{~nm}$, through blue, $430-490 \mathrm{~nm}$, blue/green, $490-510 \mathrm{~nm}$, green, $510-570 \mathrm{~nm}$, yellow, $570-600 \mathrm{~nm}$, orange, $600-620 \mathrm{~nm}$, to red, $620-700 \mathrm{~nm}$ or more. These wavelengths are approximate and the colors merge into each other. Red, such as the appearance of Congo red-stained amyloid in unpolarized light, is produced by the predominant absorption of blue/green or green, around 500 $520 \mathrm{~nm}$. Green is produced by the predominant absorption of both violet and red light, the absorption of which individually gives yellow and blue. Any green color can be matched by a suitable mixture of yellow and blue light. Absorption is measured with a spectrophotometer and is expressed as optical density, which has no units, and is given by

$$
\text { Optical density }=\log (1 / \text { transmittance }) \text {, }
$$

where

Transmittance $=($ intensity of light transmitted $)$

/(intensity of light illuminating the specimen).

At the molecular level, light is only absorbed when it is polarized parallel to a light-absorbing atomic bond. If a material is orientated so that all these bonds are parallel, appropriate wavelengths will be maximally absorbed from white light which is polarized parallel to the bonds, and the material will have its deepest color. No wavelengths will be absorbed when the light is polarized perpendicularly to the bonds, and the material should appear colorless. If this orientated material is on the specimen stage of a microscope fitted with a polarizer or an analyzer, but not both, and either the stage or the polarizing filter is rotated, the appearance of the material should change from its deepest color to colorless. This property is called dichroism, which means that either a material has different amounts of absorption of light polarized in different planes, or the material varies from colored to colorless, depending on the plane of polarization. ${ }^{4}$ The second definition follows from the first. To investigate dichroism, a soluble dye such as Congo red, which is randomly orientated in solution, can be orientated by crystallization, smearing of a drying drop on a glass slide, buffing of a dried drop, or binding to a material that itself has an orientated structure. 'Apple-green dichroism' of Congo red-stained amyloid is incorrect, because even if there is a green color, it is seen between crossed polarizer and analyzer, not when Congo red is examined with only one polarizing filter, which is the way to detect dichroism.

Strong absorption may make an orientated material appear bright against a dark background when examined between crossed polarizer and analyzer. When the material is at $45^{\circ}$ to the plane of the polarizer, polarized light is resolved into two vectors at right angles, one parallel to the absorbing bonds and the other perpendicular. After leaving the material, the vectors recombine, and the absorption in one axis may have been intense enough to make the plane of linearly polarized light rotate toward the non-absorbing axis. This rotation may allow some light to pass the crossed analyzer, and the material appears bright. This mechanism has been suggested as an explanation of the brightness of Congo red-stained amyloid between crossed polarizer and analyzer. ${ }^{5}$

\section{Birefringence, Retardance, and Compensation}

The mechanism usually said to be the explanation of this brightness is birefringence, but without further explanation of how birefringence makes something appear bright and colored. Birefringence means that a material has a range of refractive index that varies between two extremes, where

$$
\begin{aligned}
\text { Refractive index }= & (\text { velocity of light in air or a vacuum }) \\
& /(\text { velocity of light in a transmitting medium }) .
\end{aligned}
$$

Substances such as some types of glass have random arrangement of molecules and are isotropic. The refractive index is the same whatever the plane of light transmitted. Other materials, such as unstained collagen bundles, have an orientated arrangement and are anisotropic. The velocity of transmission differs between light polarized in one plane, for instance, parallel to the long axis of bundles, and light polarized at right angles. These materials have two extremes of refractive index, of which the higher indicates the slow axis of transmission and the lower indicates the fast axis. Birefringence is a property that cannot be colored apple-green or any other color, although it may be the explanation of colors. The amount of birefringence, which has no units, is the difference between the two limiting refractive indices. This can be measured by the retardance, where

$$
\begin{aligned}
\text { Retardance }= & (\text { distance travelled in air, after leaving the } \\
& \text { material,by a light wave polarized in one plane }) \\
& -(\text { distance travelled by the delayed coherent } \\
& \text { wave polarized perpendicularly }) .
\end{aligned}
$$

Coherent means that the waves are able to recombine with each other to give interference effects. Birefringence is 
retardance divided by specimen thickness, which cannot usually be measured. Retardance is a more practical measurement than birefringence and may be expressed as phase difference, by dividing linear distance by wavelength of the light. For example, a retardance of $100 \mathrm{~nm}$ in violet light of wavelength $400 \mathrm{~nm}$ is a quarter-wave phase difference.

Vectors parallel to each axis of transmission recombine after leaving a birefringent object with its axes at $45^{\circ}$ to the plane of linearly polarized light. When the retardance is nil or equals a particular wavelength, or a multiple of it, the light of that wavelength remains linearly polarized in the plane of the polarizer and cannot pass the crossed analyzer, giving a black band in the spectrum. This is destructive interference. Except at retardances of $0 \mathrm{~nm}$, this only begins to be significant at retardances approaching $400 \mathrm{~nm}$, above which in white light a series of colors is seen, called Newton's scale, as various wavelengths or combinations of them are extinguished. ${ }^{6}$ Destructive interference has been suggested as the explanation of the color of Congo red-stained amyloid between crossed polarizer and analyzer. ${ }^{7}$

For other retardances, light generally becomes elliptically polarized. The tip of the light wave, viewed end-on, now traces an ellipse and not a straight line. The direction of rotation depends upon which vector emerges first. This makes an object appear bright against the dark background, because a component of the ellipse can pass a crossed analyzer (Figure 1). As the dimension of the ellipse in the plane of the analyzer increases, more light is transmitted and the maximum is achieved when there is half-wave retardance, or

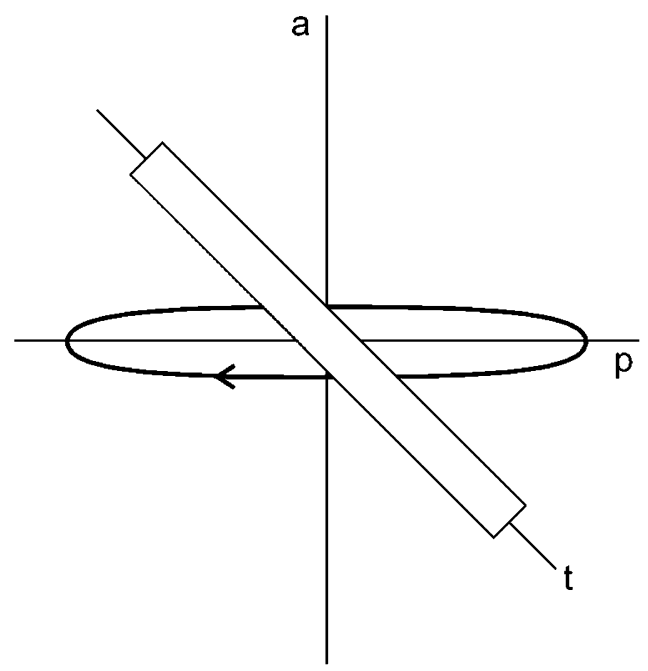

Figure 1 Production of elliptically polarized light by a birefringent object. When the object, such as an unstained collagen bundle, has an axis of transmission $(t)$ at $45^{\circ}$ between crossed polarizer $(p)$ and analyzer $(a)$, the vector of light waves in the slow axis is retarded more than the vector at right angles in the fast axis. The vectors recombine on leaving the object to give elliptically polarized light, some of which is in the plane of the analyzer, and can be transmitted, to make the object appear bright against a dark background. one wave and a half, and so on. Birefringent effects are maximal when the axes of transmission are at $45^{\circ}$ to the plane of polarization between crossed polarizer and analyzer, and they decline with rotation of object or polarizer or analyzer, disappearing when the axes are parallel and perpendicular to the plane of polarization (Figure 2).

Retardance may be measured with a compensator, so called because it compensates a phase difference, converting elliptically polarized light to linearly polarized. One type, Brace-Köhler, is a birefringent plate with known small retardance, inserted between the specimen stage and analyzer. This can measure the relatively slight retardances found in biological material and is rotated perpendicularly to the light beam to give polarized light of variable ellipticity. When the ellipse is opposite in direction of rotation and equal in dimensions to the ellipse produced by the birefringent object, linearly polarized light is produced in the plane of the polarizer, which is not transmitted by a crossed analyzer. This procedure is made with monochromatic light, meaning the light of a single wavelength, in case not all wavelengths are compensated at any setting of the compensator. Full compensation is when the object becomes maximally dark. This is difficult to judge because the background lightens as the analyzer transmits elliptically polarized light when the compensator is rotated. An easier point to detect is when intensity of object and background match, called half compensation.

\section{Dispersion of the Refractive Index, and Anomalous Colors}

For transparent materials, such as glass, the refractive index is nearly constant throughout the spectrum, showing a slight

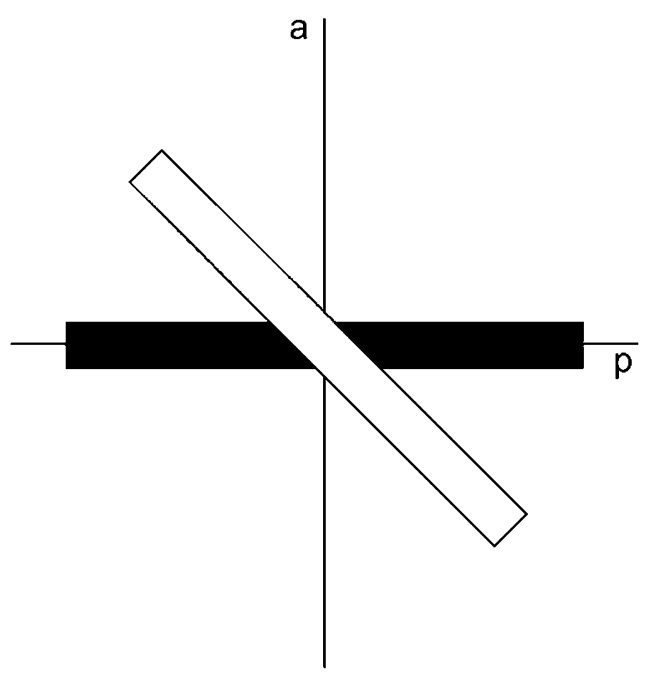

Figure 2 Effect of rotation of a birefringent object between crossed polarizer $(p)$ and analyzer $(a)$. The object, such as an unstained collagen bundle, appears maximally bright when its axes of transmission are at $45^{\circ}$ to the plane of polarization. The object appears as dark as the background when rotated to be parallel to the plane of the polarizer, as illustrated, or parallel to the plane of the analyzer. 
decline with increasing wavelength. This is normal dispersion of the refractive index, which explains the colored spectrum seen when white light passes through a triangular prism. In a birefringent material that does not absorb light, such as unstained collagen bundles, the refractive index of both axes shows normal dispersion. The birefringence is constant at every wavelength, giving white against a dark background in white light.

In contrast, any light-absorbing medium has a marked change in refractive index around an absorption peak. The refractive index falls rapidly to a minimum on the shortwave side and jumps to a maximum on the longwave side, decreasing after that, rapidly at first and then gradually as the wavelength increases. This discontinuity is called anomalous dispersion of the refractive index (Figure 3). ${ }^{1,4,6}$ In practice, damping effects within the atoms and use of narrow waveband filters that cover more than one wavelength mean that the minimum and maximum values are not achieved and that observed curves are smoother than theoretical curves. ${ }^{4}$

In an orientated, birefringent, colored material, the nonabsorbing axis shows normal dispersion, while the absorbing axis shows anomalous dispersion. The birefringence is not constant at every wavelength but varies because the difference between the refractive indices of the axes changes in relation to anomalous dispersion. The transmitted color in white light depends on the net effect of birefringence at different wavelengths, and could be anomalous, meaning a color different from that seen in unpolarized white light, not explained by Newton's scale of interference colors. ${ }^{6}$

By convention, birefringence is positive if the higher refractive index is for light polarized parallel to a feature such

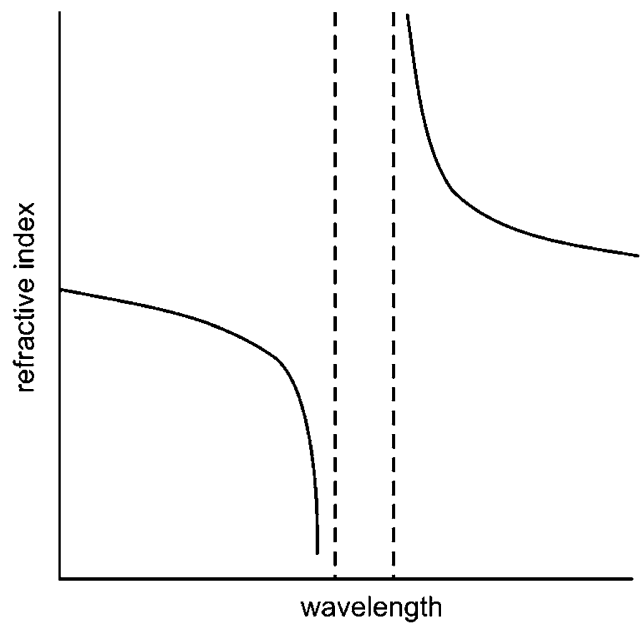

Figure 3 Anomalous dispersion of the refractive index. In a lightabsorbing medium, the refractive index changes with wavelength. Away from an absorption peak, indicated by the vertical dotted lines, in which in theory strong absorption means the refractive index cannot be measured, there is a gradual decrease in refractive index with increasing wavelength. Around the peak, there are marked changes. On the shortwave side, the index falls rapidly. On the longwave side, the index is high and falls rapidly at first. In practice, the change of refractive index on each side of the peak is less marked than shown. as long axis of a fiber or direction of smearing of a dye, and negative otherwise. A material could have positive birefringence at some wavelengths and negative at others, because in some circumstances the slow and fast axes of transmission could exchange positions. The Brace-Köhler compensator not only measures retardance but also identifies the direction of the axes of transmission.

\section{Optical Activity}

Another mechanism that has been suggested to explain the brightness of Congo red-stained amyloid between crossed polarizer and analyzer is optical activity, or optical rotation. ${ }^{8,9}$ This is rotation of the plane of linearly polarized light, independently of the plane of illumination. ${ }^{10}$ Linearly polarized light can be considered a mixture of two coherent beams of circularly polarized light, left- and right-handed. If a material has a different refractive index for the two components, there is circular birefringence, which explains optical rotation. Differential absorption of left- and right-handed circularly polarized light is circular dichroism. Optical rotatory dispersion is variation of optical rotation with wavelength. Dispersion changes markedly around an absorption peak and is called anomalous, with a change from positive to negative rotation. Combined changes in dispersion and circular dichroism around an absorption peak are the Cotton effect. Optical activity and circular dichroism typically have 1000 -fold weaker effects than linear birefringence. $^{11}$

\section{Plan of the Investigation}

Optical properties were examined of sections containing amyloid stained by Congo red, and of slides that carried Congo red orientated by smearing. These properties were investigated with either a polarizer alone or an analyzer alone, or both, with rotation of the specimen and/or a polarizing filter, to determine dichroic and birefringent effects (Figure 4). The absorption spectrum of Congo red was measured in solution, and in the dye orientated by smearing, in various conditions of polarized light. Birefringence of sections and smears was quantified by measurement of retardance at a range of wavelengths through the visible spectrum. The hypothesis was that variation of birefringence around the absorption peak of Congo red explained the observed properties.

Other suggested mechanisms were investigated. Calculation was used to assess the contribution of rotation of the plane of linearly polarized light by unequal absorption in two axes. ${ }^{5}$ Measurement of retardance showed whether this was large enough to give destructive interference. ${ }^{7}$ Optical activity and the Cotton effect would allow an analyzer to transmit light whatever was the plane of polarized light, and if these mechanisms were significant, smears or stained amyloid fibrils should never appear as dark as the background between crossed polarizer and analyzer. ${ }^{8,9}$ 


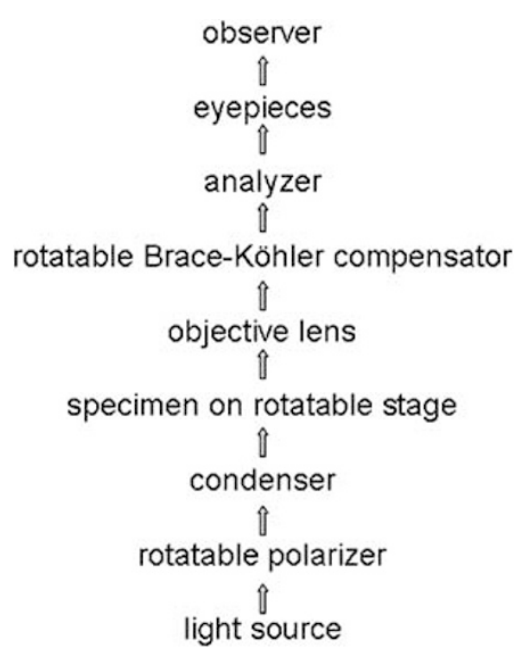

Figure 4 Arrangement of components on a microscope for polarizing microscopy. The only component not in everyday use is a Brace-Köhler compensator. Some microscopes have a rotatable analyzer and fixed polarizer, rather than vice versa. Depending on the investigation, any or all of polarizer, compensator, and analyzer may be removed from the light path.

\section{MATERIALS AND METHODS}

Most observations were on one batch of Congo red (Gurr Certistain, $343222 \mathrm{Q}$ ). Optical density of $0.5 \mathrm{~g}$ Congo red in $100 \mathrm{ml}$ distilled water, diluted $200 \times\left(3.5 \times 10^{-5} \mathrm{M}\right)$, was measured in a Shimadzu UV-160A spectrophotometer. The undiluted solution was used to make smears. Drops on glass slides, when nearly dry, were smeared with one stroke of the edge of another slide ${ }^{12}$ and examined without coverslips. Sections of kidney containing amyloid were controls for Stokes' method, ${ }^{13}$ with various slides, coverslips, and batches of dye. Optical density of smears was measured with a QDI 1000 UV-visible microspectrophotometer (CRAIC Technologies, CA, USA), with various orientations of polarizer and analyzer, sampling area $15 \mu \mathrm{m}$ square, 50 scans per measurement, measurement at every $0.33 \mathrm{~nm}$ wavelength, and open areas on slides as reference. Observations of dichroism were made on a Zeiss Axioskop microscope with rotatable stage and polarizer, plus analyzer for observations of birefringence. ${ }^{1-3}$ Retardance was measured using a BraceKöhler compensator (Leica) of retardance calibrated to range from $49.6 \mathrm{~nm}$ at wavelength $480 \mathrm{~nm}$ to $48.2 \mathrm{~nm}$ at $644 \mathrm{~nm}$. Monochromatic light was transmitted by covering the light source with narrow waveband filters that had central wavelengths mostly at $25 \mathrm{~nm}$ intervals from 400 to $700 \mathrm{~nm}$ (Glen Spectra, UK, and Chroma Technology, VT, USA). Not all filters were available for every observation. Sensitivity of polarizing microscopy was increased with maximum light intensity, minimum condenser aperture, and $\times 5$ objective. ${ }^{14}$ The Bear and Schmitt half compensation method was used, in which the compensator was rotated until intensity of object and background matched. ${ }^{2}$ Wavelengths were measured in random order, and five readings averaged per measurement. Measurements of retardance and optical density were on different smears. Retardance in nm was calculated from

$$
\begin{aligned}
\text { Retardance }= & 2 \times \text { retardance of compensator } \\
& \times \sin (2 \times \text { degrees of rotation of compensator } \\
& \text { from zero position }),
\end{aligned}
$$

and was divided by wavelength to give phase difference. This was multiplied by 360 to give degrees. These were transformed first to transmittance using the equation for an object at $45^{\circ}$ to the polarization plane,

$$
\text { Transmitted intensity } \left.=\sin ^{2} \text { (retardance } / 2\right) \text {, }
$$

and then to optical density, assuming illuminating intensity of $1.0 .^{3,5,15}$ The equation

$$
\text { Transmittance }=1 /(\text { antilog optical density })
$$

transformed optical density to transmittance. Transmittance from rotation of linearly polarized light by unequal absorption in two axes was calculated at intervals of wavelengths from

Transmitted intensity $=($ illuminating amplitude

$$
\times[1 / A-1 / B] / 2)^{2} / 2,
$$

where $A=$ antilog (optical density perpendicular to axis/2) and $B=$ antilog (optical density parallel to axis/2), assuming illuminating amplitude of 1.0 , and using optical density values for streaks at $90^{\circ}$ and $0^{\circ}$ to a polarizer. ${ }^{5}$

\section{RESULTS \\ Observations on Smears of Congo Red and Sections of Amyloid Stained by Congo Red}

Smears and sections showed dichroism, meaning that the intensity of their red color depended on the plane of polarization. In white light, without an analyzer, smears had streaks that appeared dull red when parallel to the polarizer plane and virtually colorless when perpendicular. Between these dichroic streaks were non-dichroic areas, which did not change intensity of color, where the dye had dried in random orientation, probably because tiny imperfections in the glass slides meant that there was not complete contact between them over the whole area of the drop when it was smeared (Figure $5 \mathrm{a}-\mathrm{c}$ ). In sections, the generally haphazard arrangement of amyloid made detection of dichroism difficult, but small areas where amyloid was in straight orientation were seen to change the intensity of their red color slightly with rotation of the polarizer or section.

In streaks at $45^{\circ}$ to the polarizer, with crossed analyzer, background and non-dichroic areas appeared black, and streaks mostly appeared green (Figure $5 \mathrm{~d}$ ). On rotation of the specimen, streaks stayed the same color in every quadrant, but became as dark as the background at every $90^{\circ}$, when parallel to the plane of the polarizer or analyzer. These dark positions showed that any contribution of optical activity and the Cotton effect was negligible. ${ }^{8,9}$ Streaks that were not green were near the edge of drops, and were intense red, or occasionally bright yellow or orange. These did not change 
color with rotation of the specimen but were extinguished at every $90^{\circ}$. When narrow waveband filters were inserted in the light path, transmission of red areas was nil at $550 \mathrm{~nm}$ or under, slight at $575 \mathrm{~nm}$, and marked at $600 \mathrm{~nm}$ and over. Here, the dye was thickest, with the deepest color in unpolarized light.

When examined between accurately crossed polarizer and analyzer, sections sometimes resembled thin parts of streaks. Green against black was much easier to detect than dichroic effects against white (Figure 5e). Some sections showed two colors, not uniform green, but either blue/green and yellow/ green, or blue and yellow (Figure 5f). These were in adjacent quadrants of circular structures or structures perpendicular to each other, and alternated as the slide was rotated. A few sections showed more marked changes in color on rotation, with variability in brightness of the background, generally unevenly distributed between different areas in the microscope field.

With a streak initially at $45^{\circ}$ between crossed polarizer and analyzer, rotation of either polarizer or analyzer gradually lightened the background and changed colors. One direction, opposite for polarizer and analyzer, changed green to yellow, orange, bright red, and then, when parallel to the streak, dull red (Figure 5g-i). The other direction lightened green to intense white, and then to virtually colorless when perpendicular to the streak (Figure 5j-1). Sections showed both effects in different areas and in adjacent quadrants of circular structures (Figure $5 \mathrm{~m}$ ). Rotation of the section or movement of polarizer or analyzer around the crossed position caused colors to rotate or alternate.

\section{Optical Density}

The solution of Congo red had two absorption peaks, at $345 \mathrm{~nm}$ in the ultraviolet and $499 \mathrm{~nm}$ in the blue/green. Further observations were in the visible spectrum, $400-700 \mathrm{~nm}$.

Orientated streaks examined with a polarizer, without an analyzer, had different optical density values but similar curves (Figure 6a). Optical density at $0^{\circ}$, when streaks were parallel to the polarizer, resembled that of the solution. There was only slight absorption at $90^{\circ}$, when perpendicular to the polarizer, consistent with dichroism. Optical density at $45^{\circ}$ to the polarizer was closer to that at $90^{\circ}$ than $0^{\circ}$, and differed from means of optical density values at 0 and $90^{\circ}$. Values at 0 and $90^{\circ}$, calculated at $25 \mathrm{~nm}$ intervals, were transformed to transmittance, and means were back-transformed to optical density. This gave a curve resembling optical density measured at $45^{\circ}$, showing that the transmittance at $45^{\circ}$ was halfway between that at 0 and $90^{\circ}$.

Five streaks at $45^{\circ}$ between crossed polarizer and analyzer had different optical density values but similar curves, with peaks of optical density in the violet around $420 \mathrm{~nm}$ and red at $700 \mathrm{~nm}$, and a trough in the yellow/orange at $575-600 \mathrm{~nm}$ (Figure 6b). This spectrum corresponded to the observed green color (Figure 5d).
Optical density was measured on one streak at $45^{\circ}$ to the polarizer, first with crossed analyzer, and then when the analyzer was rotated each way by 5 and $10^{\circ}$. When the analyzer was turned toward the long axis of the streak, peak optical density moved to longer wavelengths in the blue and reduced in the red, and the trough moved to longer wavelengths in the yellow/orange. The other way, optical density reduced in both violet and red, and the curve became flatter and closer to the abscissa (Figure 6c). These curves corresponded to the colors seen when the polarizer was rotated from the crossed position (Figure $5 \mathrm{~d}, \mathrm{~g}, \mathrm{~h}, \mathrm{j}$, and $\mathrm{k}$ ).

\section{Birefringence}

Retardance of 10 streaks on eight smears was measured (Figure 6d). The $400 \mathrm{~nm}$ filter hardly transmitted light, and no readings were possible. Values differed between streaks, but curves were similar, with maximum absolute values around $500 \mathrm{~nm}$. At $500 \mathrm{~nm}$, there was a change from negative retardance at shorter wavelengths to positive at longer. Measurement of six amyloid deposits with uniform, straight arrangement on three sections gave similar findings, but with generally smaller retardances (Figure 6d). This was consistent with smaller amounts of orientated Congo red in sections, on average, than in streaks. Measured retardance even on streaks never reached $100 \mathrm{~nm}$, and so destructive interference was impossible. ${ }^{7}$

The uniform green of streaks and some sections in white light between crossed polarizer and analyzer was changed to blue/green and yellow/green, or blue and yellow, by introduction and rotation of the compensator. One color at a time was seen in streaks, and pairs in sections (Figure 5n-p). On sections showing these colors between accurately crossed polarizer and analyzer without compensation, uniform green was produced by introduction and rotation of the compensator, which also darkened the background (Figure 5q).

Combinations of rotation of compensator and either polarizer or analyzer produced various colors in smears and sections. Yellow or blue revealed by compensation progressively declined as red or white progressively increased with rotation of polarizer or analyzer (Figure $5 \mathrm{r}-\mathrm{x}$ ). These colors produced singly under controlled conditions resembled those often seen in combination in the random conditions of routine practice.

Both negative and positive birefringence would produce elliptical polarization, and so transmit light. Estimated transmittance was calculated from mean absolute retardance values of the 10 streaks, and transformed to optical density. This indicated the spectral curve expected from birefringence alone from these measurements (Figure 6e). Optical density was also calculated from mean retardances of the three streaks with the highest readings at any wavelength (90.6, 93.2 and $95.4 \mathrm{~nm}$ ), which indicated the thickest streaks. This curve was closer to the abscissa, meaning that the thickest streaks would be expected to transmit more light than the average streak (Figure 6e). 
Expected transmittance from rotation of linearly polarized light by unequal absorption in two axes was transformed to optical density, and it was also added to transmittance estimated from retardance of all 10 streaks, with transformation of the sum to optical density. Rotation by unequal absorption alone was calculated to give slight transmittance, at least one log order of magnitude less than birefringence (Figure 6e). Even if effects were additive, rather than overlapping, rotation had little contribution to transmittance expected from birefringence, theoretically reducing optical density by $7 \%$ at peak transmittances around $500 \mathrm{~nm}$. The transmittance from rotation would be even less in thinner streaks than those measured, and in sections, which had less Congo red than the measured streaks. The contribution of this mechanism was negligible. ${ }^{5}$

\section{Deconstruction of Spectral Curve of Congo Red Between Crossed Polarizer and Analyzer}

Measured optical density corresponding to the green color (Figure 6b) had no resemblance to the curves calculated from retardance (Figure 6e). This showed that birefringence alone could not explain the observed spectral curve. The difference between the measured and calculated curves indicated the contribution of another factor, and resembled optical density curves of streaks examined with only a polarizer, specifically the curve at $45^{\circ}$, indicating a contribution of absorption
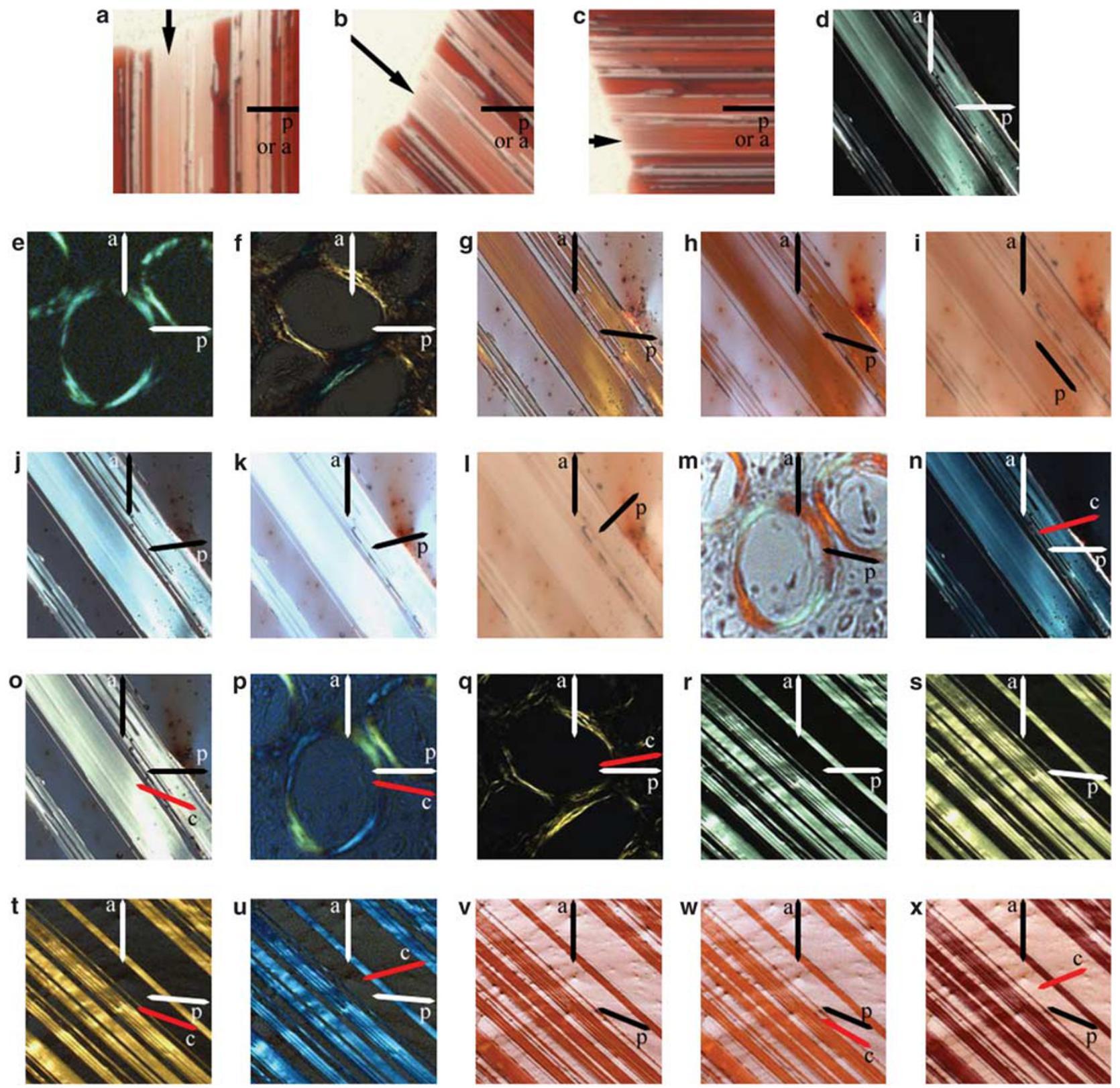
(Figure 6a). Discrepancies were a sloping tail and negative optical density at longer wavelengths for the mean of all retardances, but the discrepancies were less for the mean of the three highest retardances (Figure 6e). This suggested that the microspectrophotometric measurements had been made on generally thicker streaks than the observations of retardance.

Two further checks were made to see if birefringence and absorption together explained green. The difference in optical density between Figure $6 \mathrm{~b}$ and streaks at $45^{\circ}$ to a polarizer alone was calculated (Figure 6f). This resembled the shapes of optical density curves calculated from retardance (Figure 6e). Figure $6 \mathrm{f}$ also shows the sum of optical density expected from the three highest retardances (Figure 6e) and the measured optical density at $45^{\circ}$ to a polarizer alone (Figure $6 \mathrm{a}$ ). This was similar to the observed curve (Figure $6 \mathrm{~b}$ ). These findings are consistent with the contribution of two factors, birefringence and absorption, to the optical density spectrum of Congo red between crossed polarizer and analyzer, and so to the observed green color (Figure 5d).

\section{DISCUSSION}

We showed that polarizing effects were similar in streaks of Congo red and sections of Congo red-stained amyloid when allowances were made for the often haphazard arrangement of amyloid in tissues. From the theoretical point of view, this means that Congo red molecules must be arranged along amyloid fibrils with the light-absorbing atomic bonds all in the same orientation.

Other conclusions of the study have more practical value for pathologists. The first aim was to see whether Congo redstained amyloid between crossed polarizer and analyzer really had a pure green color, as commonly thought. The answer was that it could (Figure 5e), but even when the polarizer and analyzer were accurately crossed, there was often more than one color, especially shades of yellow/green and blue/green, or sometimes yellow and blue (Figure 5f). These alternated when the specimen was rotated. ${ }^{16}$

The next aim was to explain the green color. This is usually ascribed to birefringence, without an explanation of how this could give green. We showed that the birefringence of orientated Congo red varied with wavelength and was maximal around the absorption peak, mainly in the blue/green and green part of the spectrum (Figure 6d). ${ }^{12,17-19}$ This was explained by anomalous dispersion of the refractive index, which meant that the absorbing axis had its minimum and maximum refractive index around the peak, on the shortwave side and longwave side, respectively, while the non-absorbing axis had an almost constant refractive index, intermediate between the minimum and maximum (Figure 3). The difference between the indices of the axes was the birefringence, which had its largest absolute values around the peak.

Figure 5 Smears of Congo red and sections of kidney containing amyloid stained by Congo red, in various conditions of polarized light. The photographic images may not be accurate representations of colors seen on a microscope. Planes of polarization of polarizer $(p)$ and analyzer (a) are indicated by lines. When the Brace-Köhler compensator was introduced, the slow axis of the compensator (c) is also indicated. This compensated positive birefringence perpendicular to the direction of the slow axis, and negative birefringence parallel to the direction of the slow axis. (a-c) Three images of the same field of a smear, examined at three angles to a polarizer, without an analyzer. The same effects are obtained with an analyzer only. In (a), streaks, such as the one arrowed in each image, are almost colorless when perpendicular to the plane of polarization. There are intervening areas that are dark red and do not change color on rotation of the smear or the polarizer. These are where the dye dried haphazardly because it was not smeared by imperfections in slides. In (b), streaks at $45^{\circ}$ to the plane of polarization have a redder appearance than in (a). In (c), streaks are deepest red when parallel to the plane of polarization. This illustrates dichroism in the orientated streaks that change intensity of color, such as the one arrowed, with intervening non-orientated, non-dichroic areas. (d) Streaks aligned at $45^{\circ}$ to the plane of polarization, between crossed polarizer and analyzer. These appear green against a black background and correspond to the dichroic streaks in (a-c). This and (e) show the appearance of orientated Congo red between crossed polarizer and analyzer under ideal conditions. (e) Section between crossed polarizer and analyzer. Amyloid appears green against a black background, except at the four positions parallel to the planes of polarizer and analyzer. (f) A different section between crossed polarizer and analyzer, without any compensation. Amyloid appears yellow and blue, even though polarizer and analyzer are accurately crossed. Similar findings are common in everyday microscopy. (g-i) Identical fields showing the streaks in (d) with rotation of the polarizer toward the absorbing axis of Congo red by 5, 10, and $45^{\circ}$, respectively, giving a change to yellow in (g) orange/ red in (h) and dull red in (i). These and (j-l) mimic effects in everyday microscopy of rotation of a polarizer or analyzer from the crossed position. (j-I) Similar to $(\mathbf{g}-\mathbf{i})$, but with rotation of the polarizer by 5,10 , and $45^{\circ}$ the other way, toward the non-absorbing axis of Congo red, progressively reducing the green and increasing the white in (j, k) and giving a virtually colorless appearance in (I). ( $\mathbf{m})$ Identical field to the section in (e) with the polarizer rotated $7^{\circ}$, showing alternating quadrants of orange and pale green. This mimics an effect in everyday microscopy when polarizer and analyzer are not accurately crossed. (n, o) Identical fields to the streaks in (d) with addition of birefringence produced by a compensator, compensating positive birefringence in (n) and negative birefringence in (o) giving blue and yellow, respectively. These show that the green in (d) is produced by a mixture of blue resulting from negative birefringence $(\mathbf{n})$ and yellow from positive (o). (p) Identical field to the section in (e) with slight compensation, giving yellow/green and blue/green, with a lighter background. This mimics an effect of strain birefringence. (q) Identical field to the section in (f) with slight compensation, giving uniform green, intermediate between the yellow and blue in (f), and a darker background. This restoration of green shows that there was strain birefringence in the slide or coverslip in (f). ( $(\mathbf{r}-\mathbf{x})$ Identical fields of streaks, initially in $(\mathbf{r})$ at $45^{\circ}$ to the plane of polarization between crossed polarizer and analyzer, without compensation, appearing green, and showing in $(\mathbf{s}-\mathbf{x})$ a range of colors, produced by combinations of compensation and uncrossing of polarizer and analyzer. These mimic some colors that can be seen in everyday microscopy produced by combinations of strain birefringence and progressive rotation of polarizer or analyzer. In (s-u), the polarizer is rotated $3^{\circ}$ toward the absorbing axis of Congo red, without compensation in (s); compensating negative birefringence, adding yellow/removing blue, in (t); and compensating positive birefringence, adding blue/removing yellow, in (u). In (v-x), the polarizer is rotated $15^{\circ}$ toward the absorbing axis, without compensation in (v); compensating negative birefringence, adding yellow/removing blue, in (w); and compensating positive birefringence, adding blue/removing yellow, in (x). 
The absorbing axis was orientated parallel to streaks of Congo red and fibrils of Congo red-stained amyloid, and the non-absorbing axis was orientated perpendicularly. The birefringence accordingly changed sign, from largest negative values below the peak to largest positive above (Figure 6d). Wavelengths with the largest birefringence had the largest elliptical polarization, irrespective of sign of the birefringence,
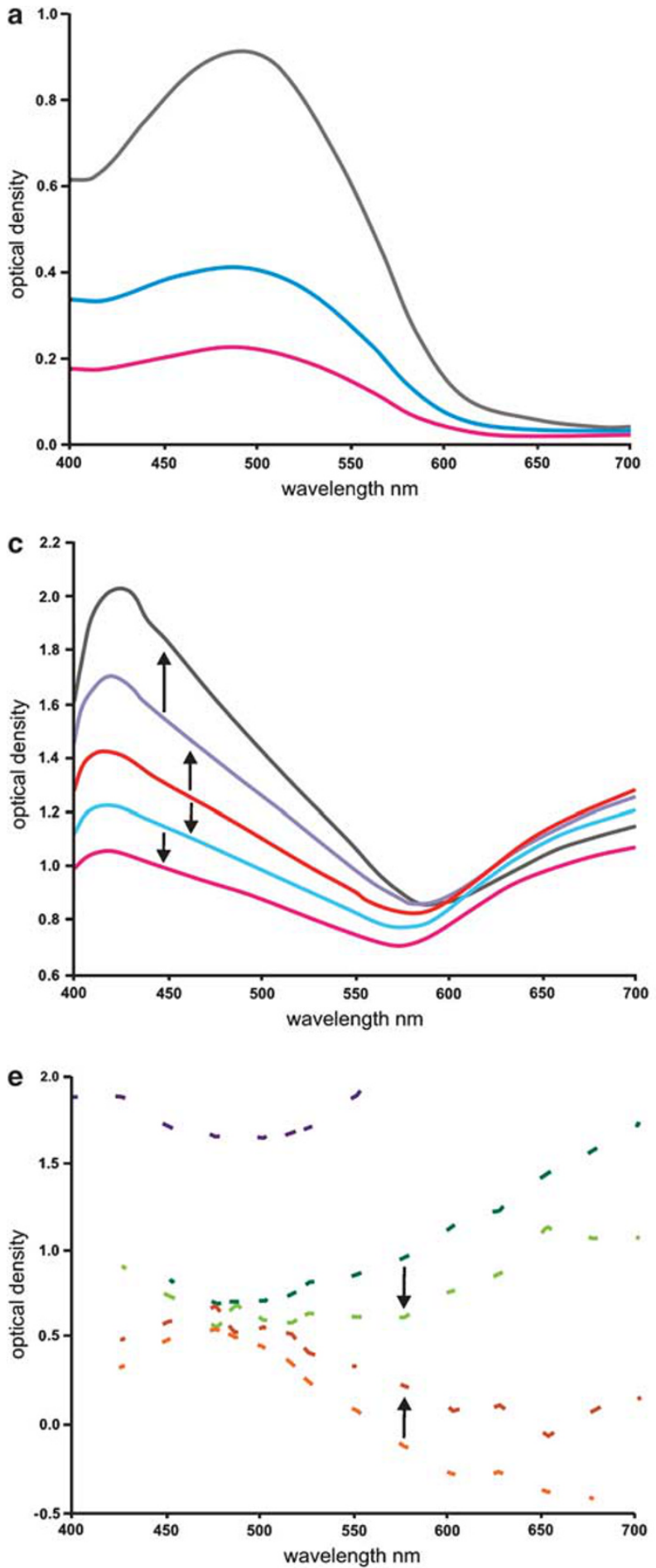

and so were transmitted with the most intensity by a crossed analyzer (Figure 1). Negative birefringence gave transmission of blue, positive gave yellow, and the observer, unable to discriminate between signs of birefringence, blended these as green (Figure $5 \mathrm{~d}, \mathrm{n}$, and o).

This is not the complete answer, because the observed spectrum of orientated Congo red between crossed polarizer
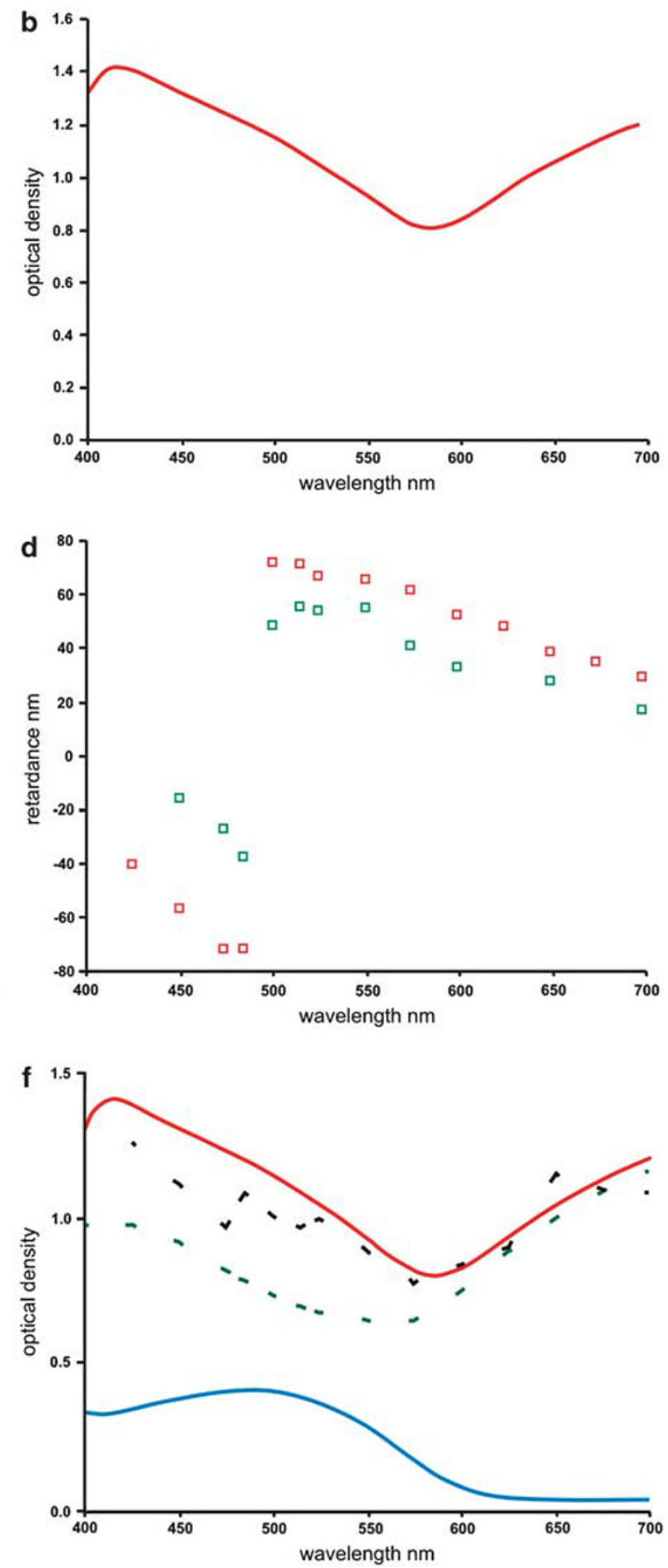
and analyzer (Figure 6b) was not the same as the spectrum expected purely from birefringence (Figure 6e). As light of the appropriate wavelengths passed through the absorbing axis, some would inevitably be absorbed. We showed that the observed spectrum was the net effect of transmission due to birefringence, and reduction of transmission by absorption (Figure 6f). The outcome was peaks of optical density in the violet and red, giving transmission of yellow and blue, which were perceived as green. In thick parts of smears, absorption predominated and eliminated the birefringence of blue, blue/green and green, leaving only transmission from birefringence of longer wavelengths such as red, as expected theoretically. ${ }^{5}$

An aim was to explain how colors other than pure green could appear, even when the polarizer and analyzer were accurately crossed. The answer is related to the change of sign of birefringence resulting from anomalous dispersion of the refractive index. Any additional birefringence in the optical system, such as that introduced by a compensator, would reduce or remove blue or yellow, if the direction of rotation of the elliptical polarization produced by the compensator was opposite to that produced by Congo red (Figure $5 n-p$ ). Unwanted additional birefringence, which may be called strain birefringence, commonly occurs in glass slides, coverslips, objective lenses, or other components on a microscope. $^{2}$ Effects of strain birefringence may be eradicated with a compensator (Figure 5q). Additional birefringence is one explanation of the multiple colors often illustrated as 'apple-green birefringence.' This also explains how colors alternate as slides are rotated, because there is a change in relative orientation of the Congo red and the additional birefringence. If strain birefringence is not uniform across the microscopic field, different colors may be seen in different areas of the field, including when the slide is rotated.

Other colors appear when the polarizer and analyzer are uncrossed, by rotation of one of them from the accurately crossed position (Figure $5 \mathrm{~g}-1$ ). The asymmetry of these effects is useful to the pathologist as a way of confirmation of amyloid, because turning a polarizing filter one way gives areas that are yellow, orange, and then red, while the other way gives bright white and then no color in the same areas. Some illustrations of 'apple-green birefringence' actually show these effects, such as those resembling Figure $5 \mathrm{~m}$.

The explanation is that with uncrossing, birefringent effects progressively decline, direct transmission and dichroic effects appear, and changing combinations of the two effects occur, while the background progressively lightens. For example, red initially appears bright from combined effects against a relatively dark background, and then pure dichroism against a light background gives dull red (Figure $5 \mathrm{~g}-\mathrm{i}$ ). The other way, as color is gradually lost, there is a stage when birefringence and dichroism give bright white against a relatively dark background from nearly equal transmission of all wavelengths (Figure $5 \mathrm{j}-\mathrm{l}$ ).

Interactions between birefringence and dichroism can be deduced from Figure $6 c$, in which the spectral curves progressively approach the two limiting dichroic curves in Figure 6a as polarizer and analyzer are uncrossed. If there is also strain birefringence, combinations of this, the usual birefringence, and dichroism give virtually any color (Figure $5 \mathrm{r}-\mathrm{x}$ ), but the pathologist now has an explanation of the variety of colors that may be seen.

Figure 6 Findings on optical density and birefringence at different wavelengths of light, in smears of Congo red and sections of kidney containing amyloid stained by Congo red. Solid lines and open dots are measured. Interrupted lines are calculated. There are changes of scale between graphs. (a) Mean optical density measured at three alignments between orientated streaks and the polarizer plane, without an analyzer: $0^{\circ}$ or parallel, uppermost (gray) line (five streaks); $45^{\circ}$, middle (blue) line (seven streaks); and $90^{\circ}$ or perpendicular, lowest (pink) line (five streaks). These confirm that orientated Congo red is dichroic, as in Figure 5a-c. (b) Mean measured optical density of five streaks aligned at $45^{\circ}$ to the plane of polarization between crossed polarizer and analyzer. This spectrum corresponds to the green in Figure $5 \mathrm{~d}$. (c) Measured optical density of a streak aligned at $45^{\circ}$ to the polarizer plane, initially between crossed polarizer and analyzer, middle (red) line. The middle spectrum corresponds to green, as in (b) and Figure $5 \mathrm{~d}$. The next line above the middle (purple) and the uppermost line (gray) show measurements with the analyzer rotated 5 and $10^{\circ}$ toward the absorbing axis of Congo red, respectively, indicated by arrows. These two spectra correspond to yellow and orange/red in Figure $5 \mathrm{~g}$ and $\mathrm{h}$. The next line below the middle (light blue) and the lowest line (pink) show measurements with the analyzer rotated by similar amounts, but in the opposite direction, indicated by arrows, away from the absorbing axis. These two spectra correspond to light green and near white in Figure $5 \mathrm{j}$ and $\mathrm{k}$. (d) Mean measured retardance of 10 streaks aligned at $45^{\circ}$ to the plane of polarization between crossed polarizer and analyzer (red), and of six areas on three sections (green). Absolute values are maximum around $500 \mathrm{~nm}$, with a change from negative retardance at shorter wavelengths to positive at longer. (e) Calculated optical density expected from retardance measurements on all 10 streaks in (d) (dark green), and from the three thickest streaks (light green), with the direction of change indicated. The uppermost (purple) line is calculated optical density from rotation of linearly polarized light by unequal absorption in two axes, which is at least one log order of magnitude more than that expected from birefringence. Also shown are lines representing subtraction from measured values for the curve in (b) of calculated optical density values derived from retardance of all 10 streaks (tan), and the three thickest (brown), with the direction of change indicated. These show the contribution of another factor, namely absorption, to birefringence, to give the observed spectrum corresponding to the green color (b). (f) Two redrawn measured curves, the upper (red) from (b) showing optical density of streaks at $45^{\circ}$ between crossed polarizer and analyzer, corresponding to the observed green color, and the lower (blue) from (a) showing optical density of streaks at $45^{\circ}$ to a polarizer only, corresponding to the absorption expected at this position. The difference between them, the green interrupted line, suggests the contribution of birefringence, and resembles the curves in (e) calculated from retardance. The black interrupted line is optical density calculated from the sum of optical density expected from the three highest retardances in (e) and the measured optical density at $45^{\circ}$ in (a). This resembles the observed curve corresponding to green, and is consistent with the combined contribution to this curve of birefringence and absorption. 
'Apple-green birefringence in polarized light' is not an appropriate phrase to describe the properties of Congo red-stained amyloid, and neither is 'apple-green dichroism.' A more accurate expression is to say that Congo red-stained amyloid, examined between crossed polarizer and analyzer, shows anomalous colors.

\section{ACKNOWLEDGEMENTS}

We are grateful to Jim Thorne, PhD, for the spectrophotometric measurements, Jackie Lewin for help with illustrations, and Steven F Quigley, PhD, Department of Physics and Astronomy, University of Birmingham, for expert advice.

\section{DISCLOSURE/DUALITY OF INTEREST}

None.

1. Brewer DB. Differences in the fine structure of collagen and reticulin as revealed by the polarising microscope. J Path Bact 1957;74:371-385.

2. Bennett HS. The microscopical investigation of biological materials with polarized light. In: Jones RM (ed). McClung's Handbook of Microscopical Technique. 3rd edn. Hafner: New York, 1967. pp 591-677.

3. Hallimond AF. The Polarizing Microscope, 3rd edn. Vickers: York, UK, 1970. pp 129-135, 257-258.

4. Born M, Wolf E. Principles of Optics. Electromagnetic Theory of Propagation, Interference and Diffraction of Light, 7 th edn. Cambridge University Press: Cambridge, UK, 1999, pp 49, 95-103, 843.

5. Goldstein DJ. Detection of dichroism with the microscope. J Microsc 1969;89:19-36.

6. Hartshorne $\mathrm{NH}$, Stuart A. Crystals and the Polarising Microscope, 4th edn. Edward Arnold: London, UK, 1970, pp 157-161, 275-281.
7. Wolman M, Bubis JJ. The cause of the green polarization color of amyloid stained with Congo red. Histochemie 1965;4:351-356.

8. Benditt EP, Eriksen N, Berglund C. Congo red dichroism with dispersed amyloid fibrils, an extrinsic Cotton effect. Proc Nat Acad Sci USA 1970;66:1044-1051.

9. Taylor $\mathrm{DL}$, Allen RD, Benditt EP. Determination of the polarization optical properties of the amyloid-Congo red complex by phase modulation microspectrophotometry. J Histochem Cytochem 1974;22:1105-1112.

10. Barron LD. Molecular Light Scattering and Optical Activity, 2nd edn. Cambridge University Press: Cambridge, UK, 2004. pp 1-10.

11. Moxon JRL, Renshaw AR. The simultaneous measurement of optical activity and circular dichroism in birefringent linearly dichroic crystal sections. I. Introduction and description of the method. J Phys Condens Matter 1990;2:6807-6836.

12. Missmahl HP. Polarisationoptischer Beitrag zur Kongorotfärbung des Amyloid. Zeit Wiss Mikrosk 1956/8;63:133-139.

13. Stokes G. An improved Congo red method for amyloid. Med Lab Sci 1976;33:79-80.

14. Swann MM, Mitchison JM. Refinements in polarized light microscopy. J Exp Biol 1950;27:226-237.

15. Glazer AM, Lewis JG, Kaminsky W. An automatic optical imaging system for birefringent media. Proc R Soc Lond A 1996:452:2751-2765.

16. Ladewig P. Double-refringence of the amyloid-Congo-red-complex in histological sections. Nature 1945;156:81-82.

17. Neubert H. Über Doppelbrechung und Dichroismus gefärbter Gele. Kolloidchemie Beiheft 1925;20:244-272.

18. Missmahl HP, Hartwig M. Polarisationoptische Untersuchungen an der Amyloidsubstanz. Virchows Arch Pathol Anat 1953;324:489-508.

19. Diezel PB, Pfleiderer A. Histochemische und polarisationische Untersuchungen am Amyloid. Virchows Arch Pathol Anat 1959;332:552-567. 